\title{
Analysis of the Atrial Signals Based on a Novel Complex Network
}

\author{
Baodan Bai* and Boyu Si \\ The college of medical instrument, Shanghai University of medicine \& health sciences, No 279, Zhouzhu Highway, Pudong \\ District, Shanghai \\ ${ }^{*}$ Corresponding author
}

\begin{abstract}
AF) is one of the most common arrhythmia in clinical, which is the major cause of embolic events and stroke, resulting in an important morbidity and mortality. The mechanisms leading to AF are still under extensive research. In this study, we present a novel complex network approach to analysis the dynamics of the heart during the whole AF process (before the $A F$ to end of the AF). Three canine models of acute AF were designed and the common parameters of the novel complex network were used to investigate the method. The results show that the novel complex network parameter can not only detect the AF, but also can estimate the vulnerability of atrial fibrillation effectively.
\end{abstract}

Keywords-atrial fibrillation; complex networt; atrial vulnerability; phase synchronization

\section{INTRODUCTION}

Atrial fibrillation (AF) is one of the most common arrhythmia in clinical, which can not only affects the quality of life of patients, serious can cause stroke, heart failure and other malignant diseases. There are about 3 million patients with permanent atrial fibrillation in the United States, and about 8 million patients in China [1][2][3]. Nevertheless, treatment of $\mathrm{AF}$ is a clinical challenge because the mechanisms of the AF are still not fully understood [4].Thus, the study of AF is very extensive. From the point of signal view, it can be divided into two major parts. One is $\mathrm{AF}$ detection and $\mathrm{AF}$ termination detection based on body surface electrocardiogram [5]. Another one is study on atrial fibrillation mechanism based on epicardium or endocardium mapping of electrical signals [6]. From the point of method, it can be divided into linear analysis method and nonlinear analysis method [7]. Previous studies have shown that nonlinear analysis methods are more suitable for the analysis of physiological systems, especially cardiac dynamic system [8].

Despite the AF has been received considerable research interest, rare attention has been paid to the AF prediction. In fact, the atrial electricity system may have changed during the transition between AF and normal sinus rhythm (NSR) [9]. It means that we can predict the vulnerability of AF with an appropriate method, and manage it timely. Thus, recently some attentions begin to pay to this point. Chen et al. proposed a phase synchronization method to study the vulnerability by analysis the sinus electrogram [10]. Zhang et al. analysis the epicardial signals correlation by Shannon Entropy [11]. Up to now, no effective method has been proposed and more researches about the atrial situation predict are needed.
In order to characterize the behaviors underlying the atrial electrical dynamics, it is necessary to take into account the global synchronization of the whole heart. Thus in this study, we proposed a novel complex network to analysis the atrial synchronization. First, by giving a step-like acetylcholine dose and electrical stimulation, acute canine AF models are established. AF vulnerability controlled by the Ladder-type acetylcholine (Ach) concentrations. Then, the phase-difference complex network was proposed to characterize the synchronization of the electrodes.

\section{METHODS}

\section{A. Experiments}

To evaluate the effective of our method, we select three healthy adult hybrids of AF are provided by the center of the animal experiment of Nanjing Gulou Hospital, China. Canine I and canine II are the male models with weight of $20 \mathrm{~kg}$ and 12 $\mathrm{kg}$ respectively; Canine III is a female model with weight of 11 $\mathrm{kg}$. In experiment, the perfusion of acetylcholine is used to induce AF in anesthetized open-chest dogs with the electrical stimulation of left atrium appendage. Accordingly, epicardial mapping system [12] with 128 unipolar electrodes is used to record electrical activities of atrial epicardial surface. Eight pieces of flexible electrode(FIGURE I A) were sutured to the right ear, left atrial appendage, right atrium posterior wall, left atrial posterior wall and four pulmonary veins, and then connect to 128 ECG mapping system(FIGURE I B).

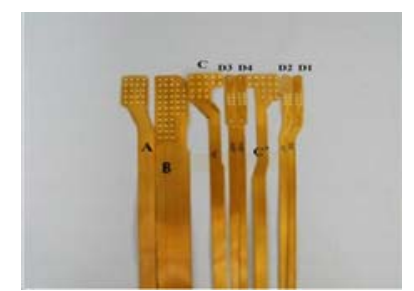

(A)

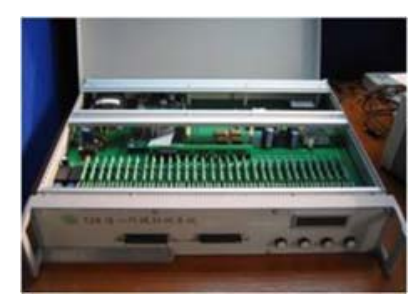

(B)
FIGURE I. EPCARDIAL MAPPING SYSTEM: (A) FLEXIBLE ELECTRODE PLAQUE (B) SYSTEM EXPERIMENT BOX

The reference electrode was sutured at the root of the aorta and connected to the reference electrode to the right ventricle. Two pairs of stimulating electrodes were sewn to the right atrial appendage and superior vena cava and right atrial junction, each pair of stimulating electrode distance of about $0.5 \mathrm{~cm}$, and connect the program stimulator. Animal intravenous intubation 
followed by micro-pump ( $2 \mathrm{~g} / \mathrm{L})$, for continuous intravenous injection of Ach $(10 \mu \mathrm{g} / \mathrm{kg} / \mathrm{min}) .3$ minutes of sinus atrial electrical signals are recorded before the electrical stimulation. Then, $20 \mathrm{~Hz}$ Burst was given to each pair of electrodes for $5 \mathrm{~s}$, with rapid stimulation of atrial fibrillation. The stimulation voltage was 2 times the pacing threshold, stimulated pulse width was $2 \mathrm{~ms}$, and the stimulus was repeated 5 times. Record the number and time of AF. And then according to the situation and duration, gradually increase the dose. 3 minutes later, repeat the above records and stimulation process. When 5 times of atrial electrical stimulation can induce 5 times persistent $\mathrm{AF}$ (AF duration $\geqslant 3 \mathrm{~min}$ ), the maximum degree of $\mathrm{AF}$ is achieved and the maximum degree of AF is defined by Ach dose (different levels of potential atrial fibrillation).

All recordings are digitized at $2000 \mathrm{~Hz}$ with 16-bit resolution. The least mean square adaptive filter is firstly used to remove the noise and ventricular artifacts. After the filtering, the sampling rate is decimated to $200 \mathrm{~Hz}$ and the mean value is subtracted from each electrode (FIGURE II).

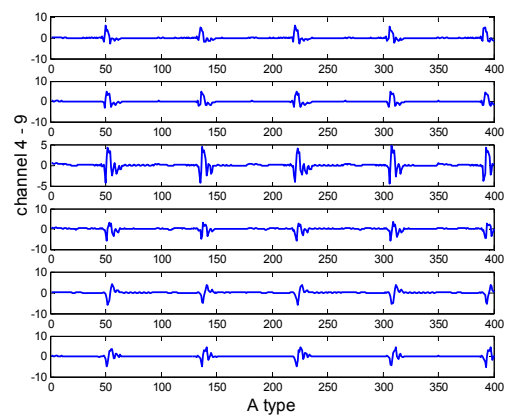

FIGURE II. RECORDED SIGNAL(A TYPE MEANS MILD AF)

\section{B. Signal Analysis}

In order to eliminate the influence of ventricular signal on data analysis, we have adopted an adaptive filtering method based on the minimum mean square error criterion. And noise is removed from the collected epicardial electrical signals.

In the last decade, the complex network becomes a popular method for the analysis of dynamical properties of real-world systems and widely applied to biological, climate networks and ecological community [13][14][15]. Among the algorithms of mapping time series into the complex networks, the recurrence complex network $[16,17]$ interprets the adjacency matrix directly from the recurrence concept in the phase space, which may provide a unifying conceptual and practical framework than others. But this method describes the relationship between the electrodes just according to the Euclidean distance in the considered phase space. In the fact, between Atrial electrical synchronization and vulnerability to AF exits a certain relationship [10]. Thus, considering those two merits, we proposed the phase-difference complex network as follows.

- The phase space vector $x_{i}(t)$ is the $i$ electrode of anterior right atrium in the time $t$. In order to achieve the balance between the network size and the cardiac cycle, each recorded signal is divided into segments whose duration is 2 seconds $(0 \leq t \leq N, N=400)$.
- The instantaneous phase $\theta_{i}(t)$ is obtained by the Hilbert transform [18] of $x_{i}(t)$. The phase difference series $\varphi_{i j}(t)$ for every two electrodes $i$ and $j$ can be calculated from $\theta_{i}(t)$ and $\theta_{j}(t)$ according to the formula (1):

$$
\varphi_{\mathrm{ij}}(\mathrm{t})=\left[\left(\theta_{\mathrm{i}}(\mathrm{t})-\theta_{\mathrm{j}}(\mathrm{t})\right)(2 \pi)^{-1}\right](1 \leq \mathrm{i}, \mathrm{j} \leq 44,1 \leq \mathrm{t} \leq \mathrm{N})
$$

- In order to reduce the computation complexity. We transform $\varphi_{i j}(t)$ to $\mathrm{S}\left(\varphi_{i j}(t)\right)$ based on symbolic method.

First, $\varphi_{i j}(t)$ was normalized to $[0,1]$. Then transformed it to $M($ here $M=5$ ) symbols by:

$S\left(\varphi_{i j}(t)\right)=\left[\mathbf{0}, 0 \leq \varphi_{i j}(t)<0.2 ; \mathbf{1}, 0.2 \leq \varphi_{i j}(t)<0.4 ; 2,0.4 \leq \varphi_{j}(t)\right.$ $\left.<0.6 ; 3,0.6 \leq \varphi_{j}(t)<0.8 ; 4,0.8 \leq \varphi_{j}(t) \leq 1\right], \quad(1 \leq i, j \leq 44,1 \leq t$

$$
\leq \bar{N}) \text {. }
$$

- To quantify the two electrodes synchronization, phase synchronization entropy (EPSI) $\rho_{i j}$ is calculated [19] by:

$$
\mathbf{\rho}_{i j}=\left(S E_{\max }-S E\right) / S E_{\max } .
$$

where, $S E_{\max }=\ln M, S E$ is Shannon entropy of the symbols. The value of EPSI $\rho_{i j}$ can describe the degree of two electrodes and is normalized to $[0,1] . \rho_{i j}$ equal to 1 means the $i$ electrode is totally synchronized with the $j$ electrode. Conversely, $\rho_{i j}$ equal to 0 means the $i$ electrode is nonsynchronous with the $j$ electrode.

- Finally, the phase difference complex network can construct based on EPSI with a threshold $r$ (here, $r=0.3$ ). Each electrode as one of the complex network node, then from the novel complex network, we can get the common complex network parameters [16]. Thus, the relationship between the vulnerability and the parameters can be discussed.

\section{RESULTS AND DISCUSSION}

In our study, through the Ach ladder-type concentrations injection and electrical stimulation, AF was observed from 20 minutes to 60 minutes. The whole process signals (previous AF signals, AF signals and post AF signals) were recorded. FIGURE III shows the matrix of the complex network of the sinus signals before AF (previous AF).
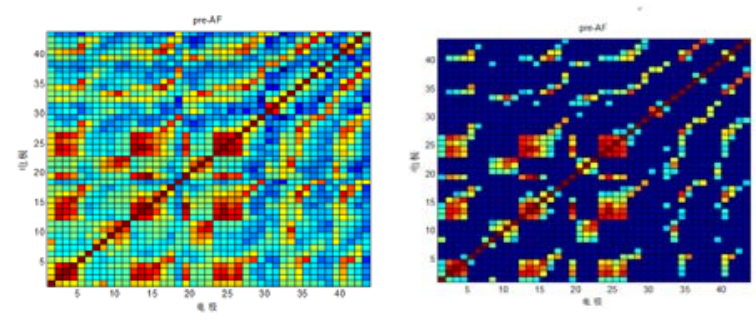

FIGURE III. THE COMPLEX NETWORK OF THE PREVIOUS AF(LEFT) AND WITH THRESHOLD (WRIGHT) AF.

FIGURE IV shows the matrix of the complex network of the AF. 


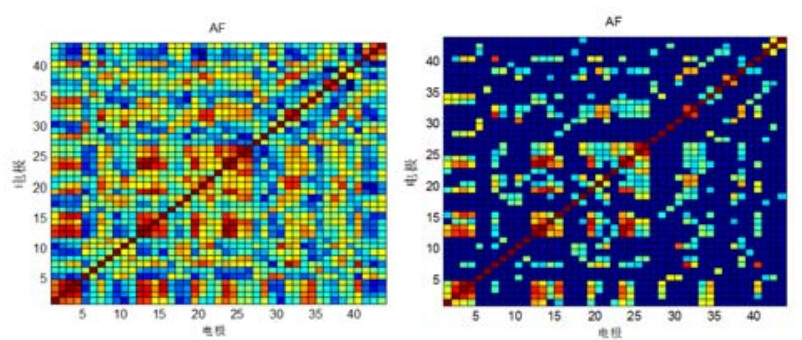

FIGURE IV. THE COMPLEX NETWORK OF THE AF(LEFT) AND WITH THRESHOLD (WRIGHT)

FIGURE V shows the complex matrix of the complex network of the sinus signals after AF (post AF).
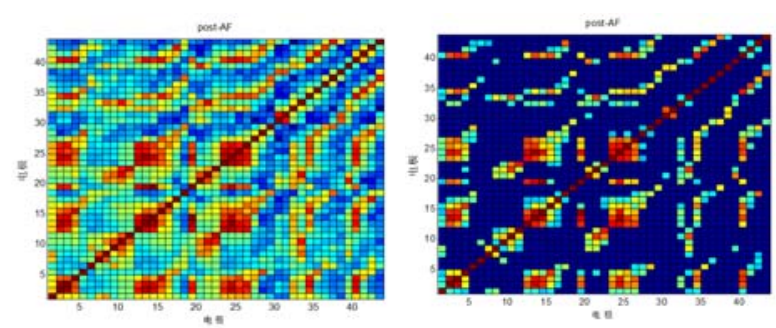

FIGURE V. THE COMPLEX NETWORK OF THE POST AF(LEFT) AND WITH THRESHOLD (WRIGHT)

FIGURE III to FIGURE V shows the complex network matrix of the whole process of the AF. Before AF, the value of EPSI is bigger thus more dots appear in the figure. That means more electrodes are synchronized in Sinus rhythm. With the occurrence of AF, the epicardial signals began to messy. The points in the matrix become discrete. That means electrodes are less synchronized during AF. And when AF finished, the electrodes synchronization ability is recovery. This result can be supported by the physiological phenomenon. The development of AF is the process of atrial electrical activity from the orderly electrical activity gradually evolved into chaotic withered waves and hence their electrical activity became nonsynchronous.

For every stage of the signal (100 samples for every stage), the statistics of the recurrence rate (REC), line distribution Shannon entropy (ENTR) and the determination of the structure (DET) are shown in TABLE I. From the table, we can see that the values of the AF are different with other two stages obviously. Thus, this novel complex network could be used to detect $\mathrm{AF}$ and to predict the $\mathrm{AF}$ determination.

TABLE I. THE PARAMETERS OF NOVEL COMPLEX NETWORK

\begin{tabular}{|l|c|c|c|}
\hline \multirow{2}{*}{$\begin{array}{c}\text { param } \\
\text { eters }\end{array}$} & \multicolumn{3}{|c|}{ Different stages } \\
\cline { 2 - 4 } REC & $0.5619 \pm 0.1415$ & $\boldsymbol{A F}$ & Post-AF \\
\hline ENTR & $0.5112 \pm 0.1775$ & $0.3652 \pm 0.2086$ & $0.4111 \pm 0.1579$ \\
\hline DET & $0.8757 \pm 0.0828$ & $0.7544 \pm 0.1826$ & $0.8463 \pm 0.1233$ \\
\hline
\end{tabular}

The vulnerability of the atrial is also discussed in this study. The FIGURE VI shows the recurrence rate of the phase difference complex network in different Ach concentrations.

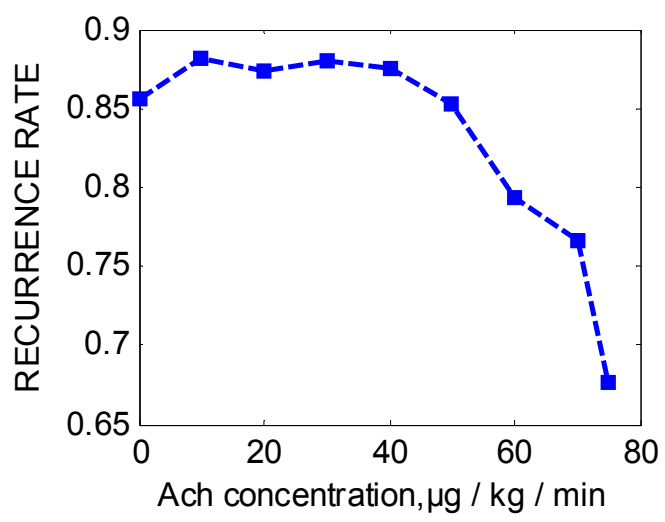

FIGURE VI. THE REC WITH DIFFERENT ACH CONCENTRATIONS

During the experiment, the state of the atrial is stable at the beginning. We cannot induce the AF when Ach concentration is less than $50 \mu \mathrm{g} / \mathrm{kg} / \mathrm{min}$. After that, as the Ach concentration increases, we can induce AF easily. There is a good consistent between the parameter and the experiment results, as shown in FIGURE VI. With the increase of the vulnerability of the atrial, the value of the REC declined. The synchronization between the electrodes becomes poor.

It is worth mentioning that recurrence rate of the novel complex network is obtained from the Sinus signals. That means we can predict the atrial situation and then realize the AF prediction.

\section{CONCLUSION}

Assuming that the normal human atrium is working synchronization controlled by the sinus node. When the AF occurs, this synchronization will appear to varying degrees of decline, leading to changes in the complexity of atrial electrical activity.

In this study, we innovate to use the EPSI depicts the electrodes synchronization. The synchronization of atrial electrical activity decreased with the number and duration of AF occurs. Combined with the advantage of the complex network for structure information, we build the complex network based on the EPSI. Our results show that there is a consistent trend between AF (duration of atrial fibrillation) and the REC of the novel complex network. Thus, the parameters of the EPSE complex network not only can detect AF and predict AF termination, but also can reflect the disorder of the atrial electrical activity effectively, and it associated with the vulnerability to AF.

\section{ACKNOWLEDGMENT}

This study was financed by the Municipal Natural Science Foundation of Shanghai (No. 16ZR1446800) and Shanghai Young Teachers Training Program for University (No. ZZJKYX15005). 


\section{REFERENCES}

[1] P.A. Wolf, J.B. Mitchell, C.S. Baker, W.B. Kannel and R.B. D'Agostino, "Impact of atrial fibrillation on mortality, stroke, and medical costs" Archives of Internal Medicine, 1998, vol.158(3), pp.229.

[2] E.J. Benjamin, P.A. Wolf, R.B. D'Agostino, H. Silbershatz, W.B.Kannel and D.Levy, "Impact of atrial fibrillation on the risk of death: the Framingham Heart Study," Circulation, 1998, vol.98(10), pp.946-952.

[3] V. Fuster, L.E.Rydén, D.S.,Cannom et al, "ACCF/AHA/HRS focused updates incorporated into the ACC/AHA/ESC 2006 guidelines for the management of patients with atrial fibrillation: a report of the American College of Cardiology Foundation/American Heart Association Task Force on practice guidelines," Journal of the American College of Cardiology, 2011, vol.57(11), pp.269-367.

[4] F. Rahman, G.F. Kwan and E.J. Benjamin, "Global epidemiology of atrial fibrillation," Nature Reviews Cardiology, 2014, vol.11(11), pp.639-654.

[5] F.Chiarugi, M.Varanini, F.Cantini, F. Conforti, G.Vrouchos, "Noninvasive ECG as a tool for predicting termination of paroxysmal atrial fibrillation," Biomedical Engineering, IEEE Transactions on, 2007 , vol. 54(8),pp.1399-1406.

[6] U. Richter, L. Faes, F. Ravelli, L. Sornmo, "Propagation pattern analysis during atrial fibrillation based on the adaptive group LASSO," Engineering in Medicine and Biology Society, Boston, 2011, pp.55355538

[7] B. Bai ,Y. Wang and C. Yang, "Predicting atrial fibrillation inducibility in a canine model by multi-threshold spectra of the recurrence complex," Medical Engineering \& Physics, 2013 , vol.35 (5) :668.

[8] L.T. Mainard, G. Calcagnini, A. Porta, F. Censi, P. Bartolini, S. Cerutti , "Linear and non-linear parameters for the classification of atrial fibrillation episodes from intra-atrial signals," Computers in Cardiology Hannover, 1999, pp.691-694

[9] C. Huang, S. Ye, H. Chen, D. L. Li, F.T. He and Y.W. Tu, "A novel method for detection of the transition between atrial fibrillation and sinus Rhythm," IEEE Transactions on Biomedical Engineering 2011,58,pp.1113-1119.

[10] Y. Chen, Z. Wu, C. Yang, et al, "Investigation of Atrial Vulnerability by Analysis of the Sinus Node EG From Atrial Fibrillation Models Using a Phase Synchronization Method," IEEE transactions on bio-medical engineering, 2012, vol.59(9),pp.2668-76.

[11] L. Zhang and C. Yang, "The Correlation Analysis of the Epicardia Signals by Shannon Entropy," Chinese Journal of Medical Instrumentation, 2014, vol.38(3),pp.165-7.

[12] C. W.Yang, W. J. Lu, T. Zhou, X.M. Wu, Z.X. Fang, "Development of epicardial mapping system for studying atrial fibrillation," International Conference on BioMedical Engineering and Informatics 2008,pp. 606609.

[13] C. S Zhou., L. Zemanova, G. Zamora and C. C. Hilgetag, "Kurths J. Hierarchical organization unveiled by functional connectivity in complex brain networks," Physical Review Letters, 2006, vol.238101,pp.1-4.

[14] J. F. Donges, Y. Zou, N. Marwan and J. Kurths, "Complex networks in climate dynamics," The European Physical Journal-Special Topics, 2009, vol.174, pp.57-179.

[15] J. A. Dunne, R. J. Williams and N. D. Martinez, "Food-web structure and network theory: the role of connectance and size," Proceeding of National Acad Sciences USA, 2002,vol.99, pp.12917-12922.

[16] C. S. Zhou, L. Zemanova, G. Zamora, C. C. Hilgetag and J. Kurth, "Recurrence networks - a novel paradigm for nonlinear time series analysis,"New Journal of Physics, 2010,vol. 033025, pp.1-41.

[17] R. V. Donner, M. Small, J. F. Dongers, N. Marwan, Y. Zou, R. Xiang and J. Kurths, "Recurrence-based time series analysis by means of complex network methods," Arxiv preprint 2010, vol.6032, pp.1-29.

[18] N. E. Huang, "New method for nonlinear and nonstationary time series analysis: empirical mode decomposition and Hilbert spectral analysis," Proceedings of SPIE - The International Society for Optical Engineering, 2000, vol.4056, pp.197-209.
[19] P.Tass, M. G. Rosenblum, J. Weule et al, "Detection of n:m Phase Locking from Noisy Data: Application to Magneto-encephalography," Physical Review Letters, 1998, vol.81(15),pp.3291-3294. 